\title{
EXTREMAL METRICS ON BLOWUPS ALONG SUBMANIFOLDS
}

\author{
REZA SEYYEDALI AND GÁBOR SZÉKELYHIDI
}

\begin{abstract}
We give conditions under which the blowup of an extremal Kähler manifold along a submanifold of codimension greater than two admits an extremal metric. This generalizes work of Arezzo-Pacard-Singer, who considered blowups in points.
\end{abstract}

\section{INTRODUCTION}

A basic question in Kähler geometry is the existence of extremal metrics on Kähler manifolds, in the sense of Calabi [4]. A Kähler metric $\omega_{M}$ on $M$ is an extremal metric if the gradient $\nabla S\left(\omega_{M}\right)$ of its scalar curvature is a holomorphic vector field on $M$. The Yau-Tian-Donaldson conjecture [26, 22, 7, 18, relates the existence of an extremal metric on a compact Kähler manifold to an algebro-geometric stability condition, but so far there are only a few existence results beyond the Kähler-Einstein case [25, 22, 5].

In this paper, following the works of Arezzo-Pacard [1, 2, Arezzo-Pacard-Singer [3] and the second author [19, 21] we investigate the existence of an extremal metric on a blowup $\mathrm{Bl}_{S} M$ of $M$ along a smooth submanifold, assuming that $M$ admits an extremal metric $\omega_{M}$. The main new feature in our work is that we allow $\operatorname{dim} S>0$, while previous works focused on blowups in points with the exception of Hashimoto [8] who considered blowups of projective spaces in lines.

In order to state our result we set up some notation. We suppose that $S$ is a codimension- $k$ submanifold of $M$, and we write $G$ for the group of Hamiltonian isometries of $\left(M, \omega_{M}\right)$. There is an associated moment map

$$
\mu: M \rightarrow \mathfrak{g}^{*},
$$

normalized so that $\langle\mu, \xi\rangle$ has zero integral for each $\xi \in \mathfrak{g}$. Denoting by $\mathscr{S}$ the space of codimension- $k$ complex submanifolds of $M$, the group $G$ acts on $\mathscr{S}$, preserving a natural symplectic form, and we have a moment map

$$
\begin{aligned}
\mu_{\mathscr{S}}: \mathscr{S} & \rightarrow \mathfrak{g}^{*}, \\
S & \mapsto \int_{S} \mu \omega_{M}^{n-k} .
\end{aligned}
$$

We identify $\mathfrak{g}=\mathfrak{g}^{*}$ using the $L^{2}$-product on Hamiltonian functions, and so we can naturally think of $\mu_{\mathscr{S}}(S)$ as a vector field on $M$. In analogy with the result in [19, in this paper we prove the following.

Theorem 1. Suppose that $S \in \mathscr{S}$ is a submanifold such that $\nabla S\left(\omega_{M}\right)$ and the vector field $\mu_{\mathscr{S}}(S)$ are tangent to $S$. Assume also that the codimension of $S$ is $k>2$. Then $\mathrm{Bl}_{S} M$ admits an extremal metric in the class $\left[\omega_{M}\right]-\epsilon^{2}[E]$ for sufficiently small $\epsilon>0$.

G.Sz. is supported in part by NSF grants DMS-1306298 and DMS-1350696. 
The strategy of the proof is very similar to that employed in [19. Because of technical difficulties we have not obtained the same result when $k=2$, although it is very likely that it is true in that case as well.

Our result can be used to obtain many new examples of extremal metrics. The simplest situation is when $\left(M, \omega_{M}\right)$ has trivial isometry group, and so in particular $\omega_{M}$ has constant scalar curvature. In this case the moment map $\mu_{\mathscr{S}}$ is trivial, and so for any submanifold $S \subset M$ of codimension greater than two the blowup $\mathrm{Bl}_{S} M$ admits a constant scalar curvature metric in $\left[\omega_{M}\right]-\epsilon^{2}[E]$ for small $\epsilon$. A more general result, allowing for a non-trivial automorphism group, analogous to [3. Theorem 2.4], is the following.

Corollary 2. Suppose that $\omega_{M}$ is an extremal metric on $M$, and let $T$ be a maximal torus in the isometry group of $\left(M, \omega_{M}\right)$. Suppose that $S \subset M$ has codimension greater than 2, and the action of $T$ preserves $S$. Then $\mathrm{Bl}_{S} M$ admits an extremal metric in $\left[\omega_{M}\right]-\epsilon^{2}[E]$ for sufficiently small $\epsilon>0$.

Proof. The vector field $J \nabla S\left(\omega_{M}\right)$ is invariant under the adjoint action of the isometry group of $\omega_{M}$, and so it is in the center of the Lie algebra $\mathfrak{g}$ of the isometry group $G$. In particular $J \nabla S\left(\omega_{M}\right) \in \mathfrak{t}$, where $\mathfrak{t}$ is the Lie algebra of $T$. Similarly because the moment map $\mu_{\mathscr{S}}$ is equivariant, $\mu_{\mathscr{S}}(S)$ is in the center of the stabilizer of $S$ under the infinitesimal action of $\mathfrak{g}$. By our assumption this stabilizer contains $\mathfrak{t}$, so any element in its center must belong to $\mathfrak{t}$. In particular $\mu_{\mathscr{S}}(S) \in \mathfrak{t}$ (here we are identifying $\mathfrak{g} \cong \mathfrak{g}^{*}$ as before), and so $\mu_{\mathscr{S}}(S)$ fixes $S$. Theorem 1 then applies.

This corollary applies for example to subspaces $\mathbf{P}^{k} \subset \mathbf{P}^{n}$ as long as $n>k+2$. In this way we obtain some extensions of the work of Hashimoto [8], who showed that $\mathrm{Bl}_{\mathbf{P}^{1}} \mathbf{P}^{n}$ admits an extremal metric for all $n$. More generally we can let $M$ be any toric manifold which admits an extremal metric, for instance a Kähler-Einstein metric obtained using the existence result of Wang-Zhu 24. We can then choose $S \subset M$ to be a toric submanifold of codimension greater than 2 .

There are also more general submanifolds $S \subset \mathbf{P}^{n}$ satisfying the assumption in Theorem 1 that $\mu_{\mathscr{S}}(S)$ is tangent to $S$. The condition $\mu_{\mathscr{S}}(S)=0$ means that $S \subset \mathbf{P}^{n}$ is a balanced embedding, and Donaldson 6 showed that if $\operatorname{Aut}(S)$ is trivial, and $S$ admits a constant scalar curvature metric, then there are balanced embeddings $S \subset \mathbf{P}^{N}$ for sufficiently large $N$. This result was generalized by the first named author [16] to the case when $S$ has non-trivial automorphisms, and admits an extremal metric (see also Mabuchi [11, 14, Hashimoto 9] for other work in this direction). As a consequence we have the following.

Corollary 3. Let $\left(S, \omega_{S}\right)$ be an extremal Kähler manifold, with $\omega_{S} \in c_{1}(L)$ for a line bundle $L \rightarrow S$. Fix an integer $r>0$, and an embedding $S \subset \mathbf{P}^{N}$ using a basis of sections of $L^{r}$. If $r$ is sufficiently large, then $\mathrm{Bl}_{S} \mathbf{P}^{N}$ admits an extremal metric in the class $\left[\omega_{F S}\right]-\epsilon^{2}[E]$ for small $\epsilon$.

Proof. Theorem 1.1 in [16] implies that under the assumptions there exist relatively balanced embeddings $S \subset \mathbf{P}^{N}$ using a basis of sections of $L^{r}$ for sufficiently large $r$. This means precisely that under these embeddings $\mu_{\mathscr{S}}(S)$, identified with a vector field on $\mathbf{P}^{N}$, is tangent to $S$. Our main result, Theorem 1 , then implies the required result.

The structure of the paper is the following. In Section 2 we will write down a metric $\omega_{\epsilon}$ on $\mathrm{Bl}_{S} M$ giving a first approximation to the metric that we are looking 
for, and we will present the main gluing result that we need to prove. In Section 3 we will show that the linearized operator of our problem is invertible. We will complete the proof of our main result in Section 4 by constructing a better approximate solution $\widetilde{\omega}_{\epsilon}$, in an analogous way to what was done in [3, 19], and then controlling the relevant non-linear terms.

\section{The GLUing PROBLEM}

2.1. A first approximate solution. Suppose as in the introduction that $\left(M, \omega_{M}\right)$ is a compact Kähler manifold such that $\omega_{M}$ is an extremal metric. Let $S \subset M$ be a codimension $k$ submanifold, where $k>2$. Our goal in this section is to construct a Kähler metric $\omega_{\epsilon}$ on the blowup $\mathrm{Bl}_{S} M$ in the class $\left[\omega_{M}\right]-\epsilon^{2}[E]$ for sufficiently small $\epsilon$, which will be a first approximation to the extremal metric that we seek. In previous work by Arezzo-Pacard 1, Arezzo-Pacard-Singer [3] and the second author [19], $S$ was a point, and the approximate solution on $\mathrm{Bl}_{S} M$ was constructed by identifying an annulus around $S$ by an annulus inside the blowup $\mathrm{Bl}_{0} \mathrm{C}^{n}$. When $S$ is a submanifold, there is no longer a standard form of a neighborhood of $S$, and so we will instead view $\mathrm{Bl}_{S} M$ as a completion of $M \backslash S$ under a suitable metric. In other words we will identify the complement of the exceptional divisor in $\mathrm{Bl}_{S} M$ with $M \backslash S$, and our constructions will primarily take place on $M \backslash S$. We then simply need to ensure that our metric extends to a smooth metric on $\mathrm{Bl}_{S} M$, which we will achieve by using the usual coordinate charts covering the blowup.

The basic building block for constructing extremal metrics on blowups is the Burns-Simanca metric [17] on $\mathrm{Bl}_{0} \mathbf{C}^{k}$. This is a scalar flat, asymptotically flat Kähler metric

$$
\eta=\sqrt{-1} \partial \bar{\partial}\left(|w|^{2}+\gamma(|w|) \log |w|^{2}+\psi\left(|w|^{2}\right)\right),
$$

where $\psi:[0, \infty)$ is smooth up to the boundary, and $\psi$ is in the weighted space $C_{2-k}^{\infty}$, i.e. $\nabla^{i} \psi(t)=O\left(t^{2-k-i}\right)$ for all $i$, as $t \rightarrow \infty$. In addition $\gamma: \mathbf{R} \rightarrow \mathbf{R}$ is a cutoff function such that $\gamma(t)=1$ for $t<1$ and $\gamma(t)=0$ for $t>2$. There are also more refined expansions of $\psi$. We will need to use that (see e.g. [19, Lemma 26])

$$
\psi\left(|w|^{2}\right)=|w|^{4-2 k}+\widetilde{\psi}\left(|w|^{2}\right),
$$

where $\widetilde{\psi} \in C_{1-k}^{\infty}$.

We will define $\omega_{\epsilon}$ by using the Kähler potential of the Burns-Simanca metric, but replacing $|w|$ by the distance function $d$ to the submanifold $S$, with respect to the metric $\omega_{M}$. Note that $d^{2}$ is a smooth function in a tubular neighborhood of $S$. For small $\epsilon>0$ let us define $r_{\epsilon}=\epsilon^{\alpha}$ for

$$
\alpha=\frac{2 k}{2 k+1} \text {. }
$$

In addition let $\gamma:[0, \infty) \rightarrow \mathbf{R}$ be a cutoff function as above, and define $\gamma_{2}: M \rightarrow \mathbf{R}$ by $\gamma_{2}=\gamma\left(r_{\epsilon}^{-1} d\right)$, and $\gamma_{1}=1-\gamma_{2}$. So $\gamma_{1}$ is supported away from $S$, while $\gamma_{2}$ is supported near $S$.

Finally we define

$$
\omega_{\epsilon}=\omega_{M}+\epsilon^{2} \sqrt{-1} \partial \bar{\partial}\left(\gamma_{2}\left[\gamma\left(\epsilon^{-1} d\right) \log \left(\epsilon^{-2} d^{2}\right)+\psi\left(\epsilon^{-2} d^{2}\right)\right]\right)
$$

on $M \backslash S$. 
Proposition 4. For sufficiently small $\epsilon$, the form $\omega_{\epsilon}$ defines a Kähler metric on $M \backslash S$, extending to a smooth metric on $\mathrm{Bl}_{S} M$.

In the proof we will need the following.

Lemma 5. At any point $p \in S$, we can choose coordinates $z_{1}, \ldots, z_{k}, w_{1}, \ldots, w_{n-k}$, defined for $|z|,|w|<1$, such that $S=\left\{z_{i}=0\right\}$ and

$$
d^{2}=|z|^{2}(1+\rho(z, w)),
$$

where $\rho=O(|z|+|w|)$, and all derivatives of $\rho$ are bounded. In addition

$$
\omega_{M}=\sqrt{-1} \partial \bar{\partial}\left(|z|^{2}+|w|^{2}+\phi(z, w)\right),
$$

where $\nabla^{i} \phi=O\left(|z|^{3-i}+|w|^{3-i}\right)$ for $i<3$, while higher order derivatives are bounded. All of these bounds can be chosen to be uniform in the point $p$.

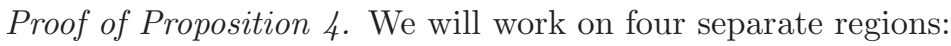

- On the set where $d>2 r_{\epsilon}$, we have $\omega_{\epsilon}=\omega_{M}$, so it is a smooth metric.

- On the set $r_{\epsilon} / 2<d<4 r_{\epsilon}$, we have contributions from the derivatives of $\gamma_{2}$, but the term involving log is not present. The asymptotics of $\psi$ imply that, measured with respect to the metric $\omega_{M}$, we have

$$
\left\|\nabla^{i}\left(\omega_{\epsilon}-\omega_{M}\right)\right\|=O\left(\epsilon^{2 k-2} r_{\epsilon}^{2-2 k-i}\right),
$$

for all $i$. It follows that for sufficiently small $\epsilon$, the form $\omega_{\epsilon}$ is also positive.

- On the set $2 \epsilon<d<r_{\epsilon}$ we have $\gamma_{2}=1$ and $\gamma\left(\epsilon^{-1} d\right)=0$. We change coordinates, using Lemma 5. In terms of $z_{1}, \ldots, z_{k}, w_{1}, \ldots, w_{n-k}$ above, we set

$$
\begin{aligned}
Z_{i} & =\epsilon^{-1} z_{i} \\
W_{j} & =\epsilon^{-1} w_{j} .
\end{aligned}
$$

By shifting the center of the coordinate system we can assume that $|W|<1$. We can compare $\epsilon^{-2} \omega_{\epsilon}$ with the product metric on $\mathrm{Bl}_{0} \mathbf{C}^{k} \times \mathbf{C}^{n-k}$. In our coordinates a Kähle potential for $\epsilon^{-2} \omega_{\epsilon}$ is given by

$$
\begin{aligned}
F= & \sum_{i}\left|Z_{i}\right|^{2}+\sum_{j}\left|W_{j}\right|^{2}+\epsilon^{-2} \phi\left(\epsilon Z_{i}, \epsilon W_{j}\right) \\
& +\psi\left(\epsilon^{-2} d^{2}\right),
\end{aligned}
$$

where $d$ is given by

$$
\epsilon^{-2} d^{2}=\left(\sum_{i}\left|Z_{i}\right|^{2}\right)\left(1+\rho\left(\epsilon Z_{i}, \epsilon W_{j}\right)\right) .
$$

At the same time the product metric has Kähler potential

$$
F_{\text {prod }}=\sum_{i}\left|Z_{i}\right|^{2}+\sum_{j}\left|W_{j}\right|^{2}+\psi\left(\sum_{i}\left|Z_{i}\right|^{2}\right),
$$

and this product metric is uniformly equivalent to the Euclidean metric in the $Z_{i}, W_{j}$ coordinates. We have

$$
F-F_{\text {prod }}=\epsilon^{-2} \phi\left(\epsilon Z_{i}, \epsilon W_{j}\right)+\psi\left(\epsilon^{-2} d^{2}\right)-\psi\left(|Z|^{2}\right) .
$$

The estimates we have for the derivatives of $\phi$ imply, that in the $Z_{i}, W_{j}$ coordinates

$$
\nabla^{i} \epsilon^{-2} \phi\left(\epsilon Z_{i}, \epsilon W_{j}\right)=O\left(\epsilon^{-2} \epsilon^{i}|\epsilon Z|^{3-i}\right)=O\left(\epsilon|Z|^{3-i}\right) .
$$


At the same time, we have

$$
\nabla^{i}\left(\epsilon^{-2} d^{2}-|Z|^{2}\right)=O\left(\epsilon|Z|^{3-i}\right),
$$

and so the decay estimates for $\psi$ imply that

$$
\nabla^{i}\left(\psi\left(\epsilon^{-2} d^{2}\right)-\psi\left(|Z|^{2}\right)\right)=O\left(\epsilon|Z|^{5-2 k-i}\right) .
$$

Since $|Z|>1$ and $5-2 k<3$, we have

$$
\left.\nabla^{i}\left(F-F_{\text {prod }}\right)=O\left(\epsilon^{i-2} d^{3-i}\right)\right),
$$

using also that $d$ is comparable to $\epsilon|Z|$. For small $\epsilon$ the form $\epsilon^{-2} \omega_{\epsilon}$ will then be a small perturbation of the product metric, since on this region $d \ll 1$.

- Finally, to examine the set where $d<2 \epsilon$ we perform a different change of coordinates. In terms of $z_{1}, \ldots, z_{k}, w_{1}, \ldots, w_{n-k}$ above, we set

$$
\begin{aligned}
v & =\epsilon^{-1} z_{k}, u_{i}=\frac{z_{i}}{z_{k}}, \text { for } i=1, \ldots, k-1 \\
w_{j}^{\prime} & =\epsilon^{-1} w_{j} \text { for } j=1, \ldots, n-k .
\end{aligned}
$$

We use this chart at points where $\left|z_{k}\right|>\frac{1}{2} \max \left\{\left|z_{1}\right|, \ldots,\left|z_{k-1}\right|\right\}$, say, and permute the coordinates appropriately at other points. On our region, once $\epsilon$ is sufficiently small, we have $\frac{d}{2}<|z|<2 d$. It follows that $|v|<2,\left|u_{i}\right|<2$, and by changing the basepoint for the coordinate system, we can assume that $\left|w^{\prime}\right|<1$. In these coordinates we have

$$
d^{2}=\epsilon^{2}|v|^{2}\left(1+\sum_{i}\left|u_{i}\right|^{2}\right)\left(1+\rho\left(\epsilon v u_{i}, \epsilon v, \epsilon w^{\prime}\right)\right) .
$$

Once again we will see that in these coordinates $\epsilon^{-2} \omega_{\epsilon}$ is well approximated by the product metric on $\mathrm{Bl}_{0} \mathbf{C}^{k} \times \mathbf{C}^{n-k}$. Indeed, in these coordinates a Kähler potential for $\epsilon^{-2} \omega_{\epsilon}$ is given by

$$
\begin{aligned}
F= & |v|^{2}+\sum_{i}|v|^{2}\left|u_{i}\right|^{2}+\sum_{j}\left|w_{j}^{\prime}\right|^{2}+\epsilon^{-2} \phi\left(\epsilon v u_{i}, \epsilon v, \epsilon w^{\prime}\right) \\
& +\gamma\left(\epsilon^{-1} d\right) \log |v|^{2}\left(1+\sum_{i}\left|u_{i}\right|^{2}\right) \\
& +\gamma\left(\epsilon^{-1} d\right) \log \left(1+\rho\left(\epsilon v u_{i}, \epsilon v, \epsilon w^{\prime}\right)\right)+\psi\left(\epsilon^{-2} d^{2}\right),
\end{aligned}
$$

while a Kähler potential for the product metric is

$$
F_{\text {prod }}=|v|^{2}+\sum_{i}|v|^{2}\left|u_{i}\right|^{2}+\sum_{j}\left|w_{j}^{\prime}\right|^{2}+\gamma(D) \log D^{2}+\psi\left(D^{2}\right),
$$

where

$$
D^{2}=|v|^{2}\left(1+\sum_{i}\left|u_{i}\right|^{2}\right) .
$$

On our region we have $D<4$, and the derivatives of $\psi$ are all bounded. It follows that

$$
\nabla^{i}\left(F-F_{\text {prod }}\right)=O(\epsilon)
$$


for all $i \geq 2$, where we are taking derivatives in the $u_{i}, v, w_{k}^{\prime}$ coordinates. In particular, once $\epsilon$ is sufficiently small, $\epsilon^{-2} \omega_{\epsilon}$ will define a smooth metric uniformly equivalent to the product metric on this set.

2.2. The gluing result. The overall strategy to proving Theorem 1 is the same as in [19. We first choose a maximal torus $T$ in the stabilizer $G_{S}$ of the submanifold $S$, and work $T$-equivariantly throughout. Let $H \subset G$ denote the centralizer of $T$, and $\overline{\mathfrak{h}}$ the Hamiltonian functions corresponding to the Lie algebra of $H$ (including the constants). So $\operatorname{dim} \overline{\mathfrak{h}}=\operatorname{dim} H+1$. Write $\overline{\mathfrak{t}} \subset \overline{\mathfrak{h}}$ for the subspace corresponding to $T$. The elements in $\overline{\mathfrak{t}}$ lift to the blowup $\mathrm{Bl}_{S} M$ in a natural way, giving Hamiltonians of holomorphic vector fields with respect to the metric $\omega_{\epsilon}$. For this note that the function $d$ is invariant under the action of $T$, and so $\omega_{\epsilon}$ is $T$-invariant.

In [19] we defined a lifting of the rest of the functions in $\overline{\mathfrak{h}}$ using cutoff functions, but here we proceed in a slightly different way, simply pulling back the functions under the blowdown map $\mathrm{Bl}_{S} M \rightarrow M$.

Definition 6. We define a map

$$
1: \overline{\mathfrak{h}} \rightarrow C^{\infty}\left(\mathrm{Bl}_{S} M\right)
$$

depending on $\epsilon$, in the following way. We fix a decomposition $\overline{\mathfrak{h}}=\overline{\mathfrak{t}} \oplus \mathfrak{h}^{\prime}$. We lift elements $g \in \overline{\mathfrak{t}}$ to $\mathrm{Bl}_{S} M$ in the natural way: if we write $\omega_{\epsilon}=\omega_{M}+\sqrt{-1} \partial \bar{\partial} A$, then on $M \backslash S$ we have

$$
\mathbf{l}(g)=g+\frac{1}{2} X(A)
$$

where $X=\nabla g$ is the holomorphic vector field corresponding to $g$. This function extends to give a smooth function on $\mathrm{Bl}_{S} M$, and it is the Hamiltonian, with respect to $\omega_{\epsilon}$, of the vector field $J X$.

For $g \in \mathfrak{h}^{\prime}$ we simply define $\mathbf{l}(g)=g$, and note that this also defines a smooth function on $\mathrm{Bl}_{S} M$, since the blowdown map $\mathrm{Bl}_{S} M \rightarrow M$ is smooth.

Given this definition, the gluing result that we need to show is the following.

Proposition 7. Suppose that $S \in \mathscr{S}$ is such that $\nabla S\left(\omega_{M}\right)$ is tangent to $S$. There are constants $\epsilon_{0}, c>0$ such that for all $\epsilon<\epsilon_{0}$ we can find $u \in C^{\infty}\left(\mathrm{Bl}_{S} M\right)^{T}$ and $f \in \overline{\mathfrak{h}}$ satisfying

$$
S\left(\omega_{\epsilon}+\sqrt{-1} \partial \bar{\partial} u\right)-\frac{1}{2} \nabla \mathbf{l}(f) \cdot \nabla u=\mathbf{l}(f) .
$$

In addition we have an expansion

$$
f=S\left(\omega_{M}\right)+\epsilon^{2 k-2}\left(\lambda+c_{m} \mu_{\mathscr{S}}(S)\right)+f_{\epsilon},
$$

where $c_{m}, \lambda$ are constants, and $\left|f_{\epsilon}\right| \leq c \epsilon^{\kappa}$ for some $\kappa>2 k-2$.

Based on this proposition, the proof of Theorem 1 is identical to the argument in [19, p. 1426]. For the reader's convenience we give the main points here.

Proof of Theorem 1, We are assuming that $\nabla S\left(\omega_{M}\right)$ and $\mu_{\mathscr{S}}(S)$ are tangent to $S$. We choose our maximal torus $T$ so that $\mu_{\mathscr{S}} \in \overline{\mathfrak{t}}$. Note that we also have $S\left(\omega_{M}\right) \in \overline{\mathfrak{t}}$ because $J \nabla S\left(\omega_{M}\right)$ is in the center of $G$. The complexification $H^{c}$ of the group $H$ acts on the space $\mathscr{S}$, and we want to show that for sufficiently small $\epsilon$ we can find an element $h \in H^{c}$ near the identity, so that Proposition 7 applied to the perturbed submanifold $h \cdot S$ yields an extremal metric on $\mathrm{Bl}_{h \cdot S} M$. 
The key point for this is that $f$, as a map from submanifolds to $\overline{\mathfrak{h}}$ can be viewed as a perturbation of a moment map, and so [19, Proposition 8] can be applied. We obtain a small perturbation $h \cdot S$ of $S$, such that when Proposition 7 is applied at $h \cdot S$, then the vector field induced by $f$ is tangent to $h \cdot S$. In particular the metric $\omega_{\epsilon}+\sqrt{-1} \partial \bar{\partial} u$ constructed in Proposition 7 will then be an extremal metric on $\mathrm{Bl}_{h \cdot S} M$. At the same time, $\mathrm{Bl}_{h \cdot S} M \cong \mathrm{Bl}_{S} M$, and so we obtain the required extremal metric on $\mathrm{Bl}_{S} M$.

\section{The Linearized PROBlem}

In this section we study the linearized problem corresponding to Equation (3). Let us denote by $C^{\infty}\left(\mathrm{Bl}_{S} M\right)_{0}^{T}$ the $T$-invariant functions $u$ on $\mathrm{Bl}_{S} M$ satisfying $\langle u, f\rangle=0$ for all $f \in \overline{\mathfrak{h}}$, where the inner product is computed using $\omega_{\epsilon}$. We will consider the linear operator

$$
\begin{aligned}
\widetilde{L}: C^{\infty}\left(\mathrm{Bl}_{S} M\right)_{0}^{T} \times \overline{\mathfrak{h}} & \rightarrow C^{\infty}\left(\mathrm{Bl}_{S} M\right)^{T}, \\
(u, f) & \mapsto L_{\omega_{\epsilon}}(u)-\frac{1}{2} \nabla \mathbf{l}(\mathbf{s}) \cdot \nabla u-\mathbf{l}(f) .
\end{aligned}
$$

Here $L_{\omega_{\epsilon}}$ denotes the linearization of the scalar curvature operator at $\omega_{\epsilon}$, i.e.

$$
S\left(\omega_{\epsilon}+\sqrt{-1} \partial \bar{\partial} u\right)=S\left(\omega_{\epsilon}\right)+L_{\omega_{\epsilon}}(u)+Q_{\omega_{\epsilon}}(u),
$$

for a suitable non-linear operator $Q_{\omega_{\epsilon}}$, and $\mathbf{s}=S\left(\omega_{M}\right)$. Recall (see e.g. 20, Section 4.1]) that we have

$$
L_{\omega_{\epsilon}}(u)=-\Delta^{2} u-R^{\bar{k} j} \partial_{j} \partial_{\bar{k}} u,
$$

in terms of the Ricci curvature $R_{j \bar{k}}$ of $\omega_{\epsilon}$. In addition we will need to relate this to the Lichnerowicz operator $\mathcal{D}^{*} \mathcal{D}$, where $\mathcal{D} u=\bar{\partial} \nabla^{1,0} u$. We have

$$
\mathcal{D}^{*} \mathcal{D} u=\Delta^{2} u+R^{\bar{k} j} \partial_{j} \partial_{\bar{k}} u+\frac{1}{2} \nabla S\left(\omega_{\epsilon}\right) \cdot \nabla u .
$$

We will show that the operator $\widetilde{L}$ is invertible, and that we can control the norm of its inverse in suitable weighted spaces.

3.1. Weighted spaces. We will next define the weighted Hölder spaces that we will use. Let us define the weight function $\tau: M \rightarrow \mathbf{R}$ by

$$
\tau(x)= \begin{cases}1 & \text { if } d(x) \geq 1 \\ d(x) & \text { if } \epsilon \leq d(x) \leq 1 \\ \epsilon & \text { if } d(x) \leq \epsilon,\end{cases}
$$

and extend it to $\mathrm{Bl}_{S} M$ by continuity. We define the weighted space $C_{\delta}^{l, \alpha}$ on $\mathrm{Bl}_{S} M$, depending on $\epsilon$, as follows. The estimate $\|f\|_{C_{\delta}^{l, \alpha}}<c$ means that for any $p \in \mathrm{Bl}_{S} M$ the $C^{l, \alpha}$-norm of $f$ on an $\omega_{M}$-ball of radius $\tau(p) / 10$ around $p$ is bounded by $c \tau(p)^{\delta}$, measured with respect to the scaled up metric $\tau(p)^{-2} \omega_{\epsilon}$.

In practice we can control these weighted norms as follows. On the region where $d>r_{\epsilon}$, the metric $\omega_{\epsilon}$ is uniformly equivalent to $\omega_{M}$, and so on this region $\|f\|_{C_{\delta}^{l}}<c$ means that

$$
\left|\nabla^{i} f\right|<c^{\prime} \tau^{\delta-i}
$$

for $i \leq l$, with the derivatives measured using $\omega_{M}$. On the region where $d<$ $2 r_{\epsilon}$, as we have seen in the proof of Proposition 4. the scaled up metric $\epsilon^{-2} \omega_{\epsilon}$ is uniformly equivalent to the product metric on $\mathrm{Bl}_{0} \mathbf{C}^{k} \times \mathbf{C}^{n-k}$. This in turn is 
uniformly equivalent, on suitable charts, with the Euclidean metric in terms of our coordinates $Z_{i}, W_{j}$, or $v, u_{i}, w_{j}^{\prime}$ from the proof of Proposition 4. It follows that in these coordinates $\|f\|_{C_{\delta}^{l}}<c$ means either

$$
\left|\nabla^{i} f\right|<c^{\prime} \epsilon^{\delta}|Z|^{\delta-i}
$$

in the $Z_{i}, W_{j}$ charts (where $\tau$ is comparable to $\epsilon|Z|$ ), or

$$
\left|\nabla^{i} f\right|<c^{\prime} \epsilon^{\delta}
$$

in the $v, u_{i}, w_{j}^{\prime}$ charts.

We also define analogous weighted spaces on $\mathbf{C}^{n-k} \times \mathrm{Bl}_{0} \mathbf{C}^{k}$ using the weight function given by $\tau(x)=1+d(x)$, where $d(x)$ is distance from the exceptional divisor in $\mathrm{Bl}_{0} \mathbf{C}^{k}$, and weighted spaces on $\left(\mathbf{C}^{k} \backslash\{0\}\right) \times \mathbf{C}^{n-k}$ using the weight function $\tau(x)=|z|$ in terms of the coordinate $z$ on the $\mathbf{C}^{k} \backslash\{0\}$ factor.

We have the following estimate of our liftings in the weighted spaces.

Lemma 8. If we have $g \in \overline{\mathfrak{h}}$ then

$$
\|\mathbf{l}(g)\|_{C_{0}^{l, \alpha}} \leq c\|g\| .
$$

Here $\|g\|$ denotes any fixed norm on the finite dimensional vector space $\overline{\mathfrak{h}}$.

Proof. Using the splitting $\overline{\mathfrak{h}}=\overline{\mathfrak{t}} \oplus \mathfrak{h}^{\prime}$ from Definition 6] if $g \in \mathfrak{h}^{\prime}$, then $\mathbf{l}(g)=g$. On the region where $d>2 \epsilon$, we certainly have

$$
\left|\nabla^{i} g\right|<c \tau^{-i}|g|
$$

for all $i$, since in fact all the derivatives are bounded uniformly, and $\tau \leq 1$.

On the region where $d<2 \epsilon$ we change coordinates to the $v, u_{i}, w_{j}^{\prime}$ from the proof of Proposition 4, and note that in terms of the local coordinates $z_{i}, w_{j}$ we have

$$
\begin{aligned}
\frac{\partial}{\partial v} & =\epsilon \frac{\partial}{\partial z_{k}}+\epsilon \sum_{i=1}^{k-1} u_{i} \frac{\partial}{\partial z_{i}} \\
\frac{\partial}{\partial u_{i}} & =\epsilon v \frac{\partial}{\partial z_{i}} \\
\frac{\partial}{\partial w_{j}^{\prime}} & =\epsilon \frac{\partial}{\partial w_{j}} .
\end{aligned}
$$

Using that $\left|u_{i}\right|,|v|<2$, we obtain the required estimate.

If $g \in \overline{\mathfrak{t}}$, then $\mathbf{l}(g)$ is defined by

$$
\mathbf{l}(g)=g+\frac{1}{2} X(A)
$$

where $X=\nabla g$, and

$$
A=\epsilon^{2} \gamma_{2}\left[\gamma\left(\epsilon^{-1} d\right) \log \left(\epsilon^{-2} d^{2}\right)+\psi\left(\epsilon^{-2} d^{2}\right)\right] .
$$

At the same time, in the coordinates $z, w$ from Lemma 5 the vector field $X$ in this case is of the form

$$
X=a_{i} \frac{\partial}{\partial z_{i}}+b_{j} \frac{\partial}{\partial w_{j}},
$$

where $a_{i}=O(|z|)$ since $X$ is tangent to $S$. Consider the region where $d<2 \epsilon$, which is the only place where the problematic $\log$ term appears. Here $\gamma_{2}=1$, and note 
that $X(d)=O(|z|)$, while $X(d) / d$ has bounded $z, w$-derivatives. It follows that on this region

$$
\nabla^{i} X(A)=O\left(\epsilon^{2}\right),
$$

which, combined with the estimate above for $g$ implies the result we need.

Note that if the vector field $X$ were not parallel to $S$, then $X(A)$ would blow up near $S$. This is our reason for lifting elements in $\mathfrak{h}^{\prime}$ in a different way.

3.2. Controlling the inverse. We will now think of our operator $\widetilde{L}$ as a map between suitable weighted spaces:

$$
\widetilde{L}: C_{\delta}^{4, \alpha}\left(\mathrm{Bl}_{S} M\right)_{0}^{T} \times \overline{\mathfrak{h}} \rightarrow C_{\delta-4}^{0, \alpha}\left(\mathrm{Bl}_{S} M\right),
$$

and our goal is the following result.

Proposition 9. For sufficiently small $\epsilon>0$ and $\delta \in(4-2 k, 0)$, the operator $\widetilde{L}$ is invertible, with a bound on its inverse $P$ independent of $\epsilon$.

We prove this result using a blowup argument, following the exposition in 20, Theorem 8.14]. We will need the following three lemmas.

Lemma 10. Define the linear operator

$$
\begin{aligned}
L_{M}(u, f) & =L_{\omega_{M}}(u)-\frac{1}{2} \nabla S\left(\omega_{M}\right) \cdot \nabla u-f \\
& =-\mathcal{D}^{*} \mathcal{D} u-f .
\end{aligned}
$$

If $L_{M}(u, f)=0$ and $u: M \backslash S \rightarrow \mathbf{R}$ is in the weighted space $\left(C_{\delta}^{4, \alpha}\right)_{0}$ with $\delta>4-2 k$, then $u, f=0$.

Proof. The restriction on the weight $\delta$ ensures that $u$ is a distributional solution of $L_{M}(u, f)=0$ on all of $M$, and in particular $u$ extends smoothly to $M$. On $M$ we have the equation $\mathcal{D}^{*} \mathcal{D} u+f=0$ and $\langle u, g\rangle=0$ for all $g \in \overline{\mathfrak{h}}$. This implies that both $f$ and $u$ vanish identically.

Lemma 11. If $u: \mathrm{Bl}_{0} \mathbf{C}^{k} \times \mathbf{C}^{n-k} \rightarrow \mathbf{R}$ is in the weighted space $C_{\delta}^{4, \alpha}$ with $\delta<0$, and $L_{0}(u)=0$, then $u=0$. Here $L_{0}$ denotes the Lichnerowicz operator on the product space.

Proof. We use an argument with the Fourier transform similar to that in MazzeoPacard [15] (see also Walpuski [23, Lemma A.1]). Let us write $u(z, w)$, where $z$ denotes the coordinate on $\mathrm{Bl}_{0} \mathbf{C}^{k}$. We have

$$
\begin{aligned}
L_{0}(u) & =\left(\Delta_{z}+\Delta_{w}\right)^{2} u+R^{i \bar{j}} u_{i \bar{j}} \\
& =\Delta_{z}^{2} u+2 \Delta_{z} \Delta_{w} u+\Delta_{w}^{2} u+R^{i \bar{j}} u_{i \bar{j}},
\end{aligned}
$$

where $\Delta_{z}, \Delta_{w}$ are the Laplacians on the two factors, and $R^{i \bar{j}}$ denotes the Ricci form of $\mathrm{Bl}_{0} \mathbf{C}^{k}$, with the indices raised. In particular the $u_{i \bar{j}}$ terms only involve derivatives on the $\mathrm{Bl}_{0} \mathbf{C}^{k}$ factor.

We take the Fourier transform of $u$ in the $w$ variable. This way we obtain a distribution $\hat{u}(z, \xi)$ on $\mathrm{Bl}_{0} \mathbf{C}^{k} \times \mathbf{C}^{n-k}$, satisfying the equation

$$
\Delta_{z}^{2} \hat{u}-2|\xi|^{2} \Delta_{z} \hat{u}+|\xi|^{4} \hat{u}+R^{i \bar{j}} \hat{u}_{i \bar{j}}=0 .
$$

In terms of the Lichnerowicz operator $L_{z}$ on $\mathrm{Bl}_{0} \mathbf{C}^{k}$ this can be written as

$$
L_{z}(\hat{u})-2|\xi|^{2} \Delta_{z} \hat{u}+|\xi|^{4} \hat{u}=0 .
$$


We claim that this implies that the distribution $\hat{u}$ is supported on the set $\{\xi=0\}$. To show this, let $g(z, \xi): \mathrm{Bl}_{0} \mathbf{C}^{k} \times \mathbf{C}^{n-k} \rightarrow \mathbf{R}$ be a smooth function with compact support away from $\{\xi=0\}$. We need to show that $\langle\hat{u}, g\rangle=0$.

For this we claim that there is a solution $h: \mathrm{Bl}_{0} \mathbf{C}^{k} \times \mathbf{C}^{n-k} \rightarrow \mathbf{R}$ of the equation

$$
L_{z} h-2|\xi|^{2} \Delta_{z} h+|\xi|^{4} h=g,
$$

with $h$ decaying faster than any negative power of $|z|$ in the $z$ direction, and with $h(z, \xi)=0$ if $|\xi|$ is sufficiently large (outside the support of $g$ ). This follows from the fact that for any fixed $\xi \neq 0$ the operator

$$
h \mapsto L_{z} h-2|\xi|^{2} \Delta_{z} h+|\xi|^{4} h
$$

on $\mathrm{Bl}_{0} \mathbf{C}^{k}$ is essentially self-adjoint, and has trivial kernel in $L^{2}$. Indeed any solution function $h$ in $L^{2}$ which is in the kernel would have to be rapidly decaying by applying Schauder estimates (on balls of radius $r / 2$ at distance $r$ from the exceptional divisor), and then an integration by parts shows that $h=0$.

We now have that

$$
\begin{aligned}
\langle\hat{u}, g\rangle & =\left\langle\hat{u}, L_{z} h-2|\xi|^{2} \Delta_{z} h+|\xi|^{4} h\right\rangle \\
& =\left\langle L_{z} \hat{u}-2|\xi|^{2} \Delta_{z} \hat{u}+|\xi|^{4} \hat{u}, h\right\rangle \\
& =0
\end{aligned}
$$

where the integration by parts is justified since $h$ is rapidly decaying in the $z$ direction, and has bounded support in the $\xi$ direction.

Now we know that $\hat{u}$ is supported on $\{\xi=0\}$, and as a result it is a linear combination of derivatives of the delta function at the origin in $\xi$ (with coefficients given by functions of $z$ ). In other words we can write

$$
\hat{u}(z, \xi)=\sum_{i=0}^{m} u_{i}(z) \delta^{(i)}(\xi),
$$

where each $\delta^{(i)}$ denotes an $i^{t h}$ derivative of the delta function at the origin. It follows that

$$
u(z, w)=\sum_{i=0}^{m} u_{i}(z) a_{i}(w),
$$

where each $a_{i}(w)$ is an $i^{t h}$ degree homogeneous polynomial in $w$. Since $u$ is bounded, only a constant polynomial can appear, and so we find that $u$ is purely a function of $z$. Since $L_{z}(u)=0$, and we are assuming that $u$ decays at infinity in the $z$ coordinate, it follows (see e.g. Arezzo-Pacard [1, Kovalev-Singer [10]) that $u=$ 0 .

Lemma 12. If $u:\left(\mathbf{C}^{k} \backslash\{0\}\right) \times \mathbf{C}^{n-k} \rightarrow \mathbf{R}$ is in the weighted space $C_{\delta}^{4, \alpha}$ with $\delta \in(4-2 k, 0)$ and $\Delta^{2} u=0$, then $u=0$.

Proof. Again a local argument shows that actually $u$ extends to a smooth function on $\mathbf{C}^{k} \times \mathbf{C}^{n-k}$, satisfying $\Delta^{2} u=0$. In addition we know that the function decays at infinity in the $\mathbf{C}^{k}$ factor. An argument identical to that in the previous lemma shows that $u=0$.

We will now use these results to prove Proposition 9 
Proof of Proposition 9. We use an argument by contradiction. We follow the exposition in Székelyhidi [20, Theorem 8.14] closely.

To emphasize the presence of the parameter $\epsilon$, we will denote by $\tilde{L}_{\epsilon}$ our operator with respect to the metric $\omega_{\epsilon}$. Our weighted spaces were defined in terms of local coordinates in which $\omega_{\epsilon}$ is uniformly equivalent to the Euclidean metric. Using the Schauder estimates in these local charts we obtain a uniform constant $C$ (independent of $\epsilon$ ), such that

$$
\|u\|_{C_{\delta}^{4, \alpha}}+\|f\| \leq C\left(\|u\|_{C_{\delta}^{0}}+\|f\|+\left\|\tilde{L}_{\epsilon}(u, f)\right\|_{C_{\delta-4}^{0, \alpha}}\right),
$$

for all $u, f$ (recall that $\delta \in(4-2 k, 0)$ ). We want to show that with a possibly larger constant, the same inequality holds without the $\|u\|_{C_{\delta}^{0}}+\|f\|$ terms on the right hand side, for sufficiently small $\epsilon$. Arguing by contradiction, let us suppose that for a sequence $\epsilon_{i} \rightarrow 0$ we have corresponding functions $u_{i}$ and $f_{i}$ satisfying

$$
\left\|u_{i}\right\|_{C_{\delta}^{0}}+\left\|f_{i}\right\|=1 \text {, but }\left\|\tilde{L}_{\epsilon}\left(u_{i}, f_{i}\right)\right\|_{C_{\delta-4}^{0, \alpha}}<\frac{1}{i},
$$

where note that the norms of $u_{i}$ are computed using $\omega_{\epsilon_{i}}$.

Using the equation (5) we can choose a subsequence of the $\left(u_{i}, f_{i}\right)$, converging to a limit $u: M \backslash S \rightarrow \mathbf{R}$, in the space $C_{\delta}^{4, \alpha^{\prime}}$ with $\alpha^{\prime}<\alpha$, and $f \in \overline{\mathfrak{h}}$. In particular we find that

$$
L_{M}(u, f)=0,
$$

where $L_{M}$ denotes the operator in Lemma 10. From Lemma 10 we have that $u, f=0$. This implies that $f_{i} \rightarrow 0$, and so up to choosing a further subsequence we can assume that

$$
\left\|L_{\epsilon}\left(u_{i}\right)-\frac{1}{2} \nabla \mathbf{l}(s) \cdot \nabla u\right\|_{C_{\delta-4}^{0, \alpha}}<\frac{1}{i}
$$

i.e. we can drop the term involving $\mathbf{l}\left(f_{i}\right)$ in the definition of $\widetilde{L}$.

We now need to examine the points $q_{i} \in \mathrm{Bl}_{S} M$, where $\tau_{i}^{-\delta} u_{i}$ achieves its maximum. Recall that $\tau_{i}$ is the weight function on $M$ (or on $\mathrm{Bl}_{S} M$ ) we defined before, with respect to $\omega_{\epsilon_{i}}$. By our assumption on $u_{i}$, we have $\left|\tau_{i}\left(q_{i}\right)^{-\delta} u_{i}\left(q_{i}\right)\right|=1$. We already know that $u_{i} \rightarrow 0$ on compact sets away from $S$, which implies that we must have $\tau_{i}\left(q_{i}\right) \rightarrow 0$. We have two cases depending on whether $\epsilon_{i}^{-1} \tau_{i}\left(q_{i}\right)$ is bounded or not.

Suppose first that for some $R>0$ we have $\epsilon_{i}^{-1} \tau_{i}\left(q_{i}\right)<R$ for all $i$. For sufficiently large $i$, the points $q_{i}$ will be in charts $z, w$ of the form considered in Lemma 5 , and by changing to $Z_{i}, W_{j}$ or $u_{i}, v, w^{\prime}$ coordinates as in the proof of Proposition 4 we can view $q_{i}$ as a point in $\mathrm{Bl}_{0} \mathbf{C}^{k} \times \mathbf{C}^{n-k}$, at distance at most $R$ from $E \times\{0\}$, where $E$ is the exceptional divisor. Moveover the pull-backs of $\epsilon_{i}^{-2} \omega_{\epsilon_{i}}$ in this chart will converge to the product metric on $\mathrm{Bl}_{0} \mathbf{C}^{k} \times \mathbf{C}^{n-k}$ on compact sets. Choosing a further subsequence we can the extract a limit $u$ of the functions $\epsilon_{i}^{-\delta} u_{i}$, locally in $C_{\delta}^{4, \alpha^{\prime}}$, where $u: \mathrm{Bl}_{0} \mathbf{C}^{k} \times \mathbf{C}^{n-k} \rightarrow \mathbf{R}$ is in the weighted space $C_{\delta}^{4, \alpha^{\prime}}$, satisfying $L_{0} u=0$. Lemma 11] implies that $u=0$, contradicting that $\left|\tau_{i}\left(q_{i}\right)^{-\delta} u_{i}\left(q_{i}\right)\right|=1$.

Finally we suppose that $\epsilon_{i}^{-1} \tau_{i}\left(q_{i}\right) \rightarrow \infty$, but $\tau_{i}\left(q_{i}\right) \rightarrow 0$. It follows that in our charts $z, w$ from Lemma $\left[5\right.$ we have $\tau\left(q_{i}\right)=|z|$, up to a bounded factor. Arguing as above, by taking a subsequence we can extract a limit of the $\tau\left(q_{i}\right)^{-\delta} u_{i}$, giving a function $u:\left(\mathbf{C}^{k} \backslash\{0\}\right) \times \mathbf{C}^{n-k} \rightarrow \mathbf{R}$, in the weighted space $C_{\delta}^{4, \alpha^{\prime}}$ satisfying $\Delta^{2} u=0$. Lemma 12 implies that $u=0$, which is a contradiction again. 
In sum we find that there is a constant $C^{\prime}$, such that for sufficiently small $\epsilon$ the estimate

$$
\|u\|_{C_{\delta}^{4, \alpha}}+\|f\| \leq C^{\prime}\left\|\tilde{L}_{\epsilon}(u, f)\right\|_{C_{\delta-4}^{0, \alpha}}
$$

holds. This shows in particular that $\tilde{L}_{\epsilon}$ has trivial kernel, and since it has index zero it must be invertible. In addition we obtain the required uniform bound on its inverse.

\section{The nOnlinear EQUation}

In this section we will solve Equation [3, which will then lead to the proof of Proposition 7

4.1. A better approximate solution. Our first task is to improve our approximate solution, in a similar way as was done in Arezzo-Pacard [2], Arezzo-PacardSinger 3] and also in [19]. For this we first modify $\omega_{M}$ on $M \backslash S$, by using a $T$-invariant solution of

with $h \in \overline{\mathfrak{h}}$ and

$$
\mathcal{D}^{*} \mathcal{D} \Gamma=h, \text { on } M \backslash S,
$$

$$
\Gamma=-d^{4-2 k}+\tilde{\Gamma}
$$

where $d$ is the distance from $S$ as before, and $\tilde{\Gamma}=O\left(d^{5-2 k}\right.$ ) (in fact we can have $O\left(d^{6-2 k-\tau}\right)$ for any small $\tau>0$, but we will not need this). Such a solution can be obtained by taking the Green's function for the Lichnerowicz operator $\mathcal{D}^{*} \mathcal{D}$, satisfying

$$
\mathcal{D}_{x}^{*} \mathcal{D}_{x} G(x, y)=-\delta_{y}+h_{y}
$$

for suitable $h_{y} \in \operatorname{ker}(\mathcal{D})$, and integrating along $S$ :

$$
\Gamma(x)=c \int_{S} G(x, y) d y,
$$

for a suitable constant $c$. We can ensure that $\Gamma$ is $T$-invariant and $h \in \overline{\mathfrak{h}}$ by averaging with the $T$-action.

In a distributional sense $\Gamma$ will then satisfy

$$
\mathcal{D}^{*} \mathcal{D} \Gamma=h-c_{m} \delta_{S} \text { on } M,
$$

where $c_{m}$ is a dimensional constant and $\delta_{S}$ is the current of integration along $S$. Integrating against $g \in \overline{\mathfrak{h}}$ we have

$$
\int_{M} g h \omega^{n}=c_{m} \int_{S} g \omega^{n-k} .
$$

It follows that under our identifications we have

$$
h=c_{m} \mu_{\mathscr{S}}(S)+\lambda,
$$

where $\lambda=\operatorname{Vol}(M)^{-1} c_{m}$ is a constant.

Recall that the potential $\psi$ for the Burns-Simanca metric has an expansion

$$
\psi\left(|w|^{2}\right)=-|w|^{4-2 k}+\tilde{\psi}\left(|w|^{2}\right),
$$

for large $|w|$, where $\tilde{\psi}\left(|w|^{2}\right)=O\left(|w|^{2-2 k}\right)$. We use this to define a new approximate solution

$$
\begin{aligned}
\tilde{\omega}_{\epsilon}=\omega_{M}+\sqrt{-1} \partial \bar{\partial}( & -\epsilon^{2 k-2} d^{4-2 k}+\gamma_{1} \epsilon^{2 k-2} \tilde{\Gamma} \\
& \left.+\gamma_{2} \epsilon^{2}\left[\gamma\left(\epsilon^{-1} d\right) \log \left(\epsilon^{-2} d^{2}\right)+\tilde{\psi}\left(\epsilon^{-2} d^{2}\right)\right]\right) .
\end{aligned}
$$


Alternatively we can also write

$$
\tilde{\omega}_{\epsilon}=\omega_{\epsilon}+\sqrt{-1} \partial \bar{\partial}\left(\epsilon^{2 k-2} \gamma_{1} \Gamma\right)
$$

in terms of our earlier approximate solution $\omega_{\epsilon}$.

We have

$$
\begin{aligned}
\left\|\epsilon^{2 k-2} \gamma_{1} \Gamma\right\|_{C_{2}^{l, \alpha}} & \leq c \epsilon^{2 k-2}\|\Gamma\|_{C_{2}^{l, \alpha}\left(M \backslash N_{r_{\epsilon}}(S)\right)} \\
& \leq c \epsilon^{2 k-2} r_{\epsilon}^{2-2 k},
\end{aligned}
$$

which tends to zero as $\epsilon \rightarrow 0$. It follows that $\tilde{\omega}_{\epsilon}$ is a small perturbation of $\omega_{\epsilon}$, and so (see [19, Proposition 20]) that the linearized operator of $\tilde{\omega}_{\epsilon}$ is a small perturbation of the linearized operator of $\omega_{\epsilon}$ for sufficiently small $\epsilon$.

4.2. The non-linear equation. As in [19, to prove Proposition 7, we would like to solve the equation

$$
S\left(\omega_{\epsilon}+\sqrt{-1} \partial \bar{\partial} u\right)-\frac{1}{2} \nabla \mathbf{l}(f) \cdot \nabla u=\mathbf{l}(f),
$$

with $u, f$ of the form

$$
\begin{aligned}
& u=\epsilon^{2 k-2} \gamma_{1} \Gamma+v \\
& f=S\left(\omega_{M}\right)+\epsilon^{2 k-2} h+g,
\end{aligned}
$$

where $\Gamma, h$ are defined in the previous subsection. Substituting these into Equation (6), we obtain

$$
\begin{aligned}
L(v)-\frac{1}{2} X(v) & -\mathbf{l}(g)=\mathbf{l}\left(S\left(\omega_{M}\right)\right)-S\left(\omega_{\epsilon}\right)-\epsilon^{2 k-2} L\left(\gamma_{1} \Gamma\right)-Q(u) \\
& +\frac{1}{2} \nabla \mathbf{l}\left(\epsilon^{2 k-2} h+g\right) \cdot \nabla u+\frac{1}{2} X\left(\epsilon^{2 k-2} \gamma_{1} \Gamma\right)+\epsilon^{2 k-2} \mathbf{l}(h),
\end{aligned}
$$

where $X=\nabla \mathbf{l}\left(S\left(\omega_{M}\right)\right)$, and $L=L_{\omega_{\epsilon}}$.

In the same way as in [19, using the inverse $P$ of the linearized operator given by Proposition 9, we can rewrite this as a fixed point problem for the operator

$$
\begin{aligned}
\mathcal{N}:\left(C_{\delta}^{4, \alpha}\right)_{0}^{T} \times \overline{\mathfrak{h}} & \rightarrow\left(C_{\delta}^{4, \alpha}\right)_{0}^{T} \times \overline{\mathfrak{h}} \\
(v, g) & \mapsto P F(v, g),
\end{aligned}
$$

where

$$
\begin{aligned}
F(v, g)= & \mathbf{l}\left(S\left(\omega_{M}\right)\right)-S\left(\omega_{\epsilon}\right)-\epsilon^{2 k-2} L\left(\gamma_{1} \Gamma\right)-Q(u) \\
& +\frac{1}{2} \nabla \mathbf{l}\left(\epsilon^{2 k-2} h+g\right) \cdot \nabla u+\frac{1}{2} X\left(\epsilon^{2 k-2} \gamma_{1} \Gamma\right)+\epsilon^{2 k-2} \mathbf{l}(h),
\end{aligned}
$$

and $u=\epsilon^{2 k-2} \gamma_{1} \Gamma+v$ as above. In addition we work with a weight $\delta \in(4-2 k, 0)$, but very close to $4-2 k$. Following the same argument as in [19], the key estimate that we need is the following.

Proposition 13. Choose $\delta \in(4-2 k, 0)$ very close to $4-2 k$. Let $r_{\epsilon}=e^{\alpha}$ with $\alpha=\frac{2 k}{2 k+1}$ as before. Then we have the estimate

$$
\|F(0,0)\|_{C_{\delta-4}^{0, \alpha}} \leq c r_{\epsilon}^{3-\delta} .
$$

Proof. Let us write $F=F(0,0)$, so we have

$$
\begin{aligned}
F & =\mathbf{l}\left(S\left(\omega_{M}\right)\right)-S\left(\omega_{\epsilon}\right)-\epsilon^{2 k-2} L\left(\gamma_{1} \Gamma\right)-Q\left(\epsilon^{2 k-2} \gamma_{1} \Gamma\right) \\
& +\frac{1}{2} \nabla \mathbf{l}\left(\epsilon^{2 k-2} h\right) \cdot \nabla\left(\epsilon^{2 k-2} \gamma_{1} \Gamma\right)+\frac{1}{2} X\left(\epsilon^{2 k-2} \gamma_{1} \Gamma\right)+\epsilon^{2 k-2} \mathbf{l}(h) .
\end{aligned}
$$


We will controll $F$ separately on the four regions $N_{\epsilon}(S), N_{r_{\epsilon}}(S) \backslash N_{\epsilon}(S), N_{2 r_{\epsilon}}(S) \backslash$ $N_{r_{\epsilon}}(S)$, and $M \backslash N_{2 r_{\epsilon}}(S)$.

- On $N_{\epsilon}(S)$ we have

$$
F=\mathbf{l}\left(S\left(\omega_{M}\right)\right)-S\left(\omega_{\epsilon}\right)+\epsilon^{2 k-2} \mathbf{l}(h) .
$$

Note that

$$
\left\|\mathbf{l}\left(S\left(\omega_{M}\right)\right)\right\|_{C_{\delta-4}^{0, \alpha}\left(N_{\epsilon}\right)} \leq \epsilon^{4-\delta}\left\|\mathbf{l}\left(S\left(\omega_{M}\right)\right)\right\|_{C_{0}^{0, \alpha}\left(N_{r_{\epsilon}}\right)} \leq c \epsilon^{4-\delta}\left\|S\left(\omega_{M}\right)\right\|,
$$

by Lemma 8 The term involving $h$ is even smaller. At the same time (2) implies that on $N_{\epsilon}(S)$ we have

$$
\left\|S\left(\omega_{\epsilon}\right)\right\|_{C_{\delta-4}^{0, \alpha}\left(N_{\epsilon}\right)} \leq c \epsilon^{4-\delta} \epsilon^{-1}=c \epsilon^{3-\delta},
$$

since $S\left(\omega_{\epsilon}\right)=\epsilon^{-2} S\left(\epsilon^{-2} \omega_{\epsilon}\right)$. In sum we obtain

$$
\|F\|_{C_{\delta-4}^{0, \alpha}\left(N_{\epsilon}\right)}<C \epsilon^{3-\delta} .
$$

- On $N_{r_{\epsilon}}(S) \backslash N_{\epsilon}(S)$ we still have

$$
F=\mathbf{l}\left(S\left(\omega_{M}\right)\right)-S\left(\omega_{\epsilon}\right)+\epsilon^{2 k-2} \mathbf{l}(h) .
$$

As above, from Lemma 8 we obtain on this region that

$$
\left\|\mathbf{l}\left(S\left(\omega_{M}\right)\right)\right\| \leq c r_{\epsilon}^{4-\delta}\left\|S\left(\omega_{M}\right)\right\|
$$

and we have an even better estimate for $h$. As for the scalar curvature of $\omega_{\epsilon}$ we now use (1), to see that

$$
\left\|S\left(\omega_{\epsilon}\right)\right\|_{C_{\delta-4}^{0, \alpha}\left(N_{r_{\epsilon}} \backslash N_{\epsilon}\right)} \leq c r_{\epsilon}^{3-\delta},
$$

and so

$$
\|F\|_{C_{\delta-4}^{0, \alpha}\left(N_{r_{\epsilon}} \backslash N_{\epsilon}\right)}<C r_{\epsilon}^{3-\delta} .
$$

- On $N:=N_{2 r_{\epsilon}}(S) \backslash N_{r_{\epsilon}}(S)$, we have, as above

$$
\left\|\mathbf{l}\left(S\left(\omega_{M}\right)\right)\right\|_{C_{\delta-4}^{0, \alpha}}(N) \leq c r_{\epsilon}^{4-\delta}\left\|\mathbf{l}\left(S\left(\omega_{M}\right)\right)\right\|_{C_{0}^{0, \alpha}} \leq c r_{\epsilon}^{4-\delta} .
$$

In addition

$$
\left\|\frac{1}{2} \nabla \mathbf{l}\left(\epsilon^{2 k-2} h\right) \cdot \nabla\left(\epsilon^{2 k-2} \gamma_{1} \Gamma\right)+\frac{1}{2} X\left(\epsilon^{2 k-2} \gamma_{1} \Gamma\right)+\epsilon^{2 k-2} \mathbf{l}(h)\right\|_{C_{\delta-4}^{0, \alpha}}(N) \leq c r_{\epsilon}^{4-\delta},
$$

since the largest term here is $\epsilon^{2 k-2} X\left(\gamma_{1} \Gamma\right)$, which contributes

$$
\epsilon^{2 k-2} r_{\epsilon}^{3-2 k} r_{\epsilon}^{4-\delta} \ll r_{\epsilon}^{4-\delta}
$$

to the norm.

So in this region, the main term to control in $F$ is

$$
S\left(\omega_{\epsilon}\right)+L\left(\epsilon^{2 k-2} \gamma_{1} \Gamma\right)+Q\left(\epsilon^{2 k-2} \gamma_{1} \Gamma\right)=S\left(\tilde{\omega}_{\epsilon}\right) .
$$

Using coordinates $(z, w)$, we have $\tilde{\omega}_{\epsilon}=\omega_{E}+\sqrt{-1} \partial \bar{\partial} H$, where

$$
\omega_{E}=\sqrt{-1} \partial \bar{\partial}\left(|z|^{2}+|w|^{2}\right)
$$

is the flat metric in these coordinates and

$$
H=\phi(z, w)-\epsilon^{2 k-2}|z|^{4-2 k}(1+\rho(z, w))^{4-2 k}+\epsilon^{2 k-2} \gamma_{1} \tilde{\Gamma}+\gamma_{2} \epsilon^{2} \tilde{\psi}\left(\epsilon^{-2} d^{2}\right) .
$$

Note that

$$
\nabla^{2} H=O\left(r_{\epsilon}+\epsilon^{2 k-2} r_{\epsilon}^{2-2 k}+\epsilon^{2 k} r_{\epsilon}^{-2 k}\right) \rightarrow 0 \text { as } \epsilon \rightarrow 0 .
$$


The dominant term above is $\epsilon^{2 k-2} r_{\epsilon}^{2-2 k}$, using our choice of $\alpha$. Therefore comparing $\tilde{\omega}_{\epsilon}$ to the flat metric, we get that on this region (using Lemma 21 in [19])

$$
\begin{aligned}
\left\|S\left(\tilde{\omega}_{\epsilon}\right)-\Delta_{0}^{2} H\right\|_{C_{\delta-4}^{0, \alpha}(N)} & \leq C r_{\epsilon}^{4-\delta}\left(\left\|\nabla^{2} H\right\|_{C^{2, \alpha}(N)}\left\|\nabla^{4} H\right\|_{C^{0, \alpha}(N)}\right) \\
& \leq C r_{\epsilon}^{4-\delta} \epsilon^{4 k-4} r_{\epsilon}^{2-4 k} \leq C r_{\epsilon}^{3-\delta} .
\end{aligned}
$$

On the other hand $\Delta_{0}^{2}\left(|z|^{4-2 k}\right)=0$. Hence

$$
\left\|\Delta_{0}^{2} H\right\|_{C_{\delta-4}^{0, \alpha}(N)} \leq C r_{\epsilon}^{4-\delta}\left(1+\epsilon^{2 k-2} r_{\epsilon}^{1-2 k}\right)=O\left(r_{\epsilon}^{3-\delta}\right),
$$

using again our choice of $\alpha$.

- Finally, on $M \backslash N_{2 r_{\epsilon}}(S)$ we have $\omega_{\epsilon}=\omega_{M}$, and

$$
L(\Gamma)-\frac{1}{2} X(\Gamma)=h,
$$

so

$$
F=-Q\left(\epsilon^{2 k-2} \Gamma\right)+\frac{1}{2} \nabla \mathbf{l}\left(\epsilon^{2 k-2} h\right) \cdot \nabla\left(\epsilon^{2 k-2} \Gamma\right) .
$$

Therefore, we get

$$
\begin{aligned}
\|F\|_{C_{\delta-4}^{0, \alpha}\left(M \backslash N_{2 r_{\epsilon}}\right)} & \left.\leq\left\|Q\left(\epsilon^{2 k-2} \Gamma\right)\right\|_{C_{\delta-4}^{0, \alpha}\left(M \backslash N_{2 r_{\epsilon}}\right)}+c \epsilon^{4 k-4}\|l(h)\|_{C_{1}^{1, \alpha}\|\nabla \Gamma\|_{C_{\delta-4}^{0, \alpha}\left(M \backslash N_{2 r_{\epsilon}}\right)}}\right) \\
& \leq c \epsilon^{4 k-4}\left(\|\Gamma\|_{C_{\delta}^{4, \alpha}\left(M \backslash N_{2 r_{\epsilon}}\right)}\|\Gamma\|_{C_{2}^{4, \alpha}\left(M \backslash N_{2 r_{\epsilon}}\right)}+r_{\epsilon}^{-1}\|\Gamma\|_{C_{\delta-4}^{1, \alpha}\left(M \backslash N_{2 r_{\epsilon}}\right)}\right) \\
& \leq c \epsilon^{4 k-4}\left(r_{\epsilon}^{4-2 k-\delta} r_{\epsilon}^{2-2 k}+r_{\epsilon}^{-1}\right) \\
& \leq c \epsilon^{4 k-4} r_{\epsilon}^{6-4 k-\delta}=O\left(r_{\epsilon}^{3-\delta}\right),
\end{aligned}
$$

using the choice of $\alpha$. We have used [19, Proposition 25] and the fact that $\left\|\gamma_{1} \Gamma\right\|_{C_{p}^{4, \alpha}} \leq c r_{\epsilon}^{4-2 k-p}$ for $p \geq 4-2 k$ and $\left\|\gamma_{1} \Gamma\right\|_{C_{p}^{4, \alpha}} \leq c$ for $p \leq 4-2 k$.

The following result has the same proof as Lemma 23 in [19.

Lemma 14. There is a constant $c_{1}>0$ such that if

$$
\left\|v_{i}\right\|_{C_{2}^{4, \alpha}},\left\|g_{i}\right\| \leq c_{1}
$$

then

$$
\left\|\mathcal{N}\left(v_{1}, g_{1}\right)-\mathcal{N}\left(v_{2}, g_{2}\right)\right\|_{C_{\delta}^{4, \alpha}} \leq \frac{1}{2}\left\|\left(v_{1}, g_{1}\right)-\left(v_{2}, g_{2}\right)\right\|_{C_{\delta}^{4, \alpha}}
$$

where $\|(v, g)\|_{C_{\delta}^{4, \alpha}}:=\|v\|_{C_{\delta}^{4, \alpha}}+\|g\|$

We now define the open set

$$
\mathcal{U}=\left\{(v, g) \in\left(C_{\delta}^{4, \alpha}\right)_{0}^{T} \times \overline{\mathfrak{h}}:\|(v, g)\|_{C_{\delta}^{4, \alpha}} \leq 2 C r_{\epsilon}^{3-\delta}\right\},
$$

where the constant $C$ is the uniform bound on the inverse operators $P$ (c.f. Proposition 9). We then have

Proposition 15. Suppose $\delta<0$ is sufficiently close to $4-2 k$. Then for $\epsilon>0$ sufficiently small, the map $\mathcal{N}: \mathcal{U} \rightarrow \mathcal{U}$ is a contraction, and therefore has a fixed point $\left(v_{\epsilon}, g_{\epsilon}\right)$. Moreover, $\left|g_{\epsilon}\right| \leq c \epsilon^{\kappa}$ for some $\kappa>2 k-2$. 
Proof. First note that if $(v, g) \in \mathcal{U}$, then we have

$$
\|v\|_{C_{2}^{4, \alpha}} \leq \epsilon^{\delta-2}\|v\|_{C_{\delta}^{4, \alpha}} \leq 2 C \epsilon^{\delta-2} r_{\epsilon}^{3-\delta} \leq c_{1},
$$

for sufficiently small $\epsilon$. Therefore, Lemma 14 applies on $\mathcal{U}$. We only need to check $\mathcal{N}(\mathcal{U}) \subset \mathcal{U}$. For this we have

$$
\begin{aligned}
\|\mathcal{N}(v, g)\|_{C_{\delta}^{4, \alpha}} & \leq\|\mathcal{N}(v, g)-\mathcal{N}(0,0)\|_{C_{\delta}^{4, \alpha}}+\|\mathcal{N}(0,0)\|_{C_{\delta}^{4, \alpha}} \\
& \leq \frac{1}{2}\|(v, g)\|_{C_{\delta}^{4, \alpha}}+\|\mathcal{N}(0,0)\|_{C_{\delta}^{4, \alpha}} \\
& \leq C r_{\epsilon}^{3-\delta}+C\|F(0,0)\|_{C_{\delta-4}^{0, \alpha}} \\
& \leq 2 C r_{\epsilon}^{3-\delta} .
\end{aligned}
$$

Therefore we obtain a fixed point $\left(v_{\epsilon}, g_{\epsilon}\right)$, with $\left|g_{\epsilon}\right| \leq 2 C r_{\epsilon}^{3-\delta}$. In addition our choice of $\alpha$ ensures that when $\delta$ is sufficiently close to $4-2 k$, then $\left|g_{\epsilon}\right| \leq c \epsilon^{\kappa}$, for some $\kappa>2 k-2$.

The fixed point $\left(v_{\epsilon}, g_{\epsilon}\right)$ gives the solution of (6) Proposition 5.

Acknowledgements. The authors are grateful to Michael Singer, and Rafe Mazzeo for helpful suggestions.

\section{REFERENCES}

[1] Arezzo, C., and Pacard, F. Blowing up and desingularizing constant scalar curvature Kähler manifolds. Acta Math. 196, 2 (2006), 179-228.

[2] Arezzo, C., And PACARD, F. Blowing up Kähler manifolds with constant scalar curvature II. Ann. of Math. (2) 170, 2 (2009), 685-738.

[3] Arezzo, C., Pacard, F., And Singer, M. A. Extremal metrics on blow ups. Duke Math. J. $15 \%, 1$ (2011), 1-51.

[4] Calabi, E. Extremal Kähler metrics. In Seminar on Differential Geometry, S. T. Yau, Ed. Princeton, 1982.

[5] Chen, X., Donaldson, S., and Sun, S. Kähler-Einstein metrics and stability. Int. Math. Res. Not. IMRN (2014), 2119-2125.

[6] Donaldson, S. K. Scalar curvature and projective embeddings, I. J. Differential Geom. 59 (2001), 479-522.

[7] Donaldson, S. K. Scalar curvature and stability of toric varieties. J. Differential Geom. 62 (2002), 289-349.

[8] Налнімото, Y. Stability and canonical metrics on projective spaces blown up along a line. arXiv:1508.02637

[9] Hashimoto, Y., Quantisation of extremal Kähler metrics. arXiv:1508.02643

[10] Kovalev, A., And Singer, M. Gluing theorems for complete anti-self-dual spaces. Geom. Funct. Anal. 11, 6 (2001), 1229-1281.

[11] Mabuchi, T. An obstruction to asymptotic semistability and approximate critical metrics. Osaka J. Math. 41, 2 (2004), 463-472.

[12] Mabuchi, T. Stability of extremal Kähler manifolds. Osaka J. Math. 41, 3 (2004), 563-582.

[13] Mabuchi, T. An energy-theoretic approach to theHitchin-Kobayashi correspondence for manifolds, I. Invent. Math. 159, 2 (2005), 225-243.

[14] Mabuchi, T. An energy-theoretic approach to theHitchin-Kobayashi correspondence for manifolds, II. Osaka J. Math. 46, 1 (2009), 115-139.

[15] Mazzeo, R., And PaCard, F. A construction of singular solutions for a semilinear elliptic equation using asymptotic analysis. J. Differential Geom. 44, 2 (1996), 331-370.

[16] Seyyedali, R. Relative Chow stability and extremal metrics. arXiv:1610.07555

[17] Simanca, S. R. Kähler metrics of constant scalar curvature on bundles over $C \mathrm{P}_{n-1}$. Math. Ann. 291, 2 (1991), 239-246. 
[18] SzÉKelyhidi, G. Extremal metrics and K-stability. Bull. Lond. Math. Soc. 39, 1 (2007), 76-84.

[19] SzÉkelyhidi, G. On blowing up extremal Kähler manifolds. Duke Math. J. 161, 8 (2012), $1411-1453$.

[20] SzÉKelyhidi, G. An introduction to extremal Kähler metrics, vol. 152 of Graduate Studies in Mathematics. American Mathematical Society, Providence, RI, 2014.

[21] SzéKelyhidi, G. Blowing up extremal Kähler manifolds II. Invent. Math. 200, 3 (2015), 925-977.

[22] Tian, G. Kähler-Einstein metrics with positive scalar curvature. Invent. Math. 137 (1997), $1-37$.

[23] Walpuski, T. $G_{2}$-instantons on generalised Kummer constructions. Geom. Top. 17 (2013), $2345-2388$.

[24] Wang, X.-J., And ZhU, X. Kähler-Ricci solitons on toric manifolds with positive first Chern class. Adv. Math. 188 (2004), 87-103.

[25] YAu, S.-T. On the Ricci curvature of compact Kähler manifolds and the complex MongeAmpère equation I. Comm. Pure Appl. Math. 31 (1978), 339-411.

[26] Yau, S.-T. Open problems in geometry. Proc. Symposia Pure Math. 54 (1993), 1-28.

Department of Mathematics, Boyd Graduate Studies Research Center, University of Georgia, Athens, GA 30602

E-mail address: rseyyeda@uga.edu

Department of Mathematics, University of Notre Dame, Notre Dame, IN 46556

E-mail address: gszekely@nd.edu 